\title{
Simultaneous Optimization of Start Up Scheme and Pipe Network for Multi-source Water Injection System Based on Improved Genetic Particle Swarm Optimization Algorithm
}

\author{
Jianjun YANG ${ }^{\mathrm{a}}$, Zhiyuan ZHANG ${ }^{\mathrm{b}}$, Qingtang $\mathrm{LI}^{\mathrm{c}}$ \\ School of Mechanical Engineering, Qingdao Technological University, Qingdao, 266520, China \\ aemail: yjjdem@163.com, bemail: 925771904@qq.com, cemail: 476347505@qq.com
}

\begin{abstract}
Keywords: Multi-source and Looped Water Injection System; Pump Start Up Scheme; Pipe Network Optimization; Simultaneous Optimization; Genetic Particle Swarm Optimization Algorithm; Generalized Chromosome
\end{abstract}

\begin{abstract}
In order to improve the system operating efficiency furthest, the simultaneous optimization of pump start up scheme and pipe network is necessary. Aiming at the multi-source and looped water injection system, the mathematical model for simultaneous optimization is established, in which the operating energy consumption and investment are served as objective function, the start-up and stop status, flow rate of pump, and the connection status, diameter of pipeline are taken as design variables. Aiming at the features of the simultaneous optimization problem, a dual generalized chromosome coding was proposed, the binary coding, real number coding, and integer coding are combined together. In order to improve the local searching ability, the particle swarm optimization algorithm is combined with the genetic algorithm. The method of initial solutions creating and many crossover and mutation methods that adapted to the optimize problem are designed. The example shows that the algorithm is efficient.
\end{abstract}

\section{Introduction}

Water injection system is an important power consumer in oilfield production, at present the system has developed into multi-source and looped pipe network system, and the operating status is complicated, the improvements of operating efficiency and design level of pumping station and pipe network are very important to reduce the cost. The pump efficiency and pipe network efficiency are important components of running efficiency of the system. The existing research only optimize one kind of problems in operation and pipe network optimization, for example, operation parameter optimization [1] [2] and start up scheme optimization [3] [4] are aimed at pump optimization, pipe diameter optimization [5] [6] and pipe network layout optimization [7] [8] are aimed at pipe network optimization. But the pump operating status and pipe network status are influenced each other, in order to improve the system operating efficiency furthest, the simultaneous optimization of pump operating and pipe network is necessary. Geen [9] adopted the harmony search algorithm to optimize the pump operating and pipe network in the single source pipe network, but only the outlet pressure of pump station and the pipe diameter are optimized. Wu and Simpson [10] adopted multiobjective genetic algorithm to optimize the pump configuration and the pipe diameter. In the new-built, reconstructed and expanding process of multi-source water injection system, it is necessary to optimize the start up scheme and pipe network simultaneously, optimize the start-up and stop status, operation parameters of pumps, and the pipe network layout, pipe diameter simultaneously.

\section{The Optimal Mathematical Model}

In the mathematical model, the start-up and stop status, flow rate of pump, and the connection status, diameter of pipeline are taken as design variables, the energy consumption and investment are served as objective functions. 
$\min f_{1}=\gamma \sum_{i=1}^{N_{\mathrm{p}}} \delta_{i} \frac{H_{i} Q_{i}}{\eta_{\mathrm{p} i} \eta_{\mathrm{m} i}}$

$\min f_{2}=\sum_{i=1}^{N_{\mathrm{L}}} \xi_{i} L_{i} C_{i}\left(D_{i}\right)$

In the type: $f_{1}$ is the energy consumption of the system, $\gamma$ is unit conversion factor, $N_{\mathrm{p}}$ is the total number of the pump, $H_{i} 、 Q_{i} 、 \eta_{\mathrm{p} i}$ are the head, flow rate and efficiency of pump $i$, respectively, $\eta_{\mathrm{m} i}$ is the efficiency of drive motor for pump $i$, it has little change, can be regard as constant, $\delta_{i}$ is the start-up or stop status of pump $i$, the value is 1 when it was start-up, otherwise the value is $0, f_{2}$ is the investment of pipe network, $N_{\mathrm{L}}$ is the total number of pipelines in preliminary connection graph of pipe network, $\xi_{i}$ is connection status of the pipeline $i$, the value is 1 when it was connection, otherwise the value is $0, L_{i}$ is the length of pipeline $i, C_{i}$ is the unit cost of pipeline $i$, correspond to the pipe diameter.

And the linear weighting method is adopted to transform the two objectives into single objective. That is

$$
\min f_{3}=f_{1}+\omega f_{2}
$$

In the type, $f_{3}$ is the final objective function, $\omega$ is the weight coefficient.

The following constraints should be considered.

The first constraint is water balance constraint. The sum of flow rates of all pumps is a fixed value, and equal to the total water requirement of the system.

The second set of constraints is hydraulic equilibrium constraint. When the system is running, the equilibrium equation must be met between the flow rate and pressure for each junction node, and the hydraulic equilibrium constraints are satisfied automatically in the process of simulation [4]. The node pressure values are relative values that relative to the reference node, if all of the node pressure values increase or decrease a unified value, the equilibrium equations still can be met.

The third set of constraints is pump flow rate constraint. In order to make the pump running in high efficiency area, pump flow rate should be limited within the range $\left[Q_{i \min }, Q_{i \max }\right] . Q_{i \min }, Q_{i \max }$ are the minimum and maximum flow rate of pump $i$ respectively.

The fourth set of constraints is pump working pressure constraint. In order to make the pump running normally, the pump outlet pressure should be greater than the node pressure of water injection station. The pressure difference is called pump-pipe pressure difference. After a set of pump flow rates are given, using the equilibrium equation, a set of node pressure values that relative to the reference node can be obtained. After the unified adjustment of the node pressure values, each pump outlet pressure can be greater than the node pressure of water injection station, and the pump-pipe pressure difference should be kept as small as possible at the same time. Because there is friction loss, we set the minimum value of pump-pipe pressure difference is $0.05 \mathrm{MPa}$. So the pump working pressure constraint is satisfied.

The fifth set of constraints is node pressure constraint. The pressure value of each consumption node should be not less than the minimum working pressure.

$$
p_{i} \geq p_{i \min }
$$

In the type, $p_{i}$ is the calculation pressure value of node $i, p_{i \text { min }}$ is minimum pressure required at node $i$.

The sixth set of constraints is pipe diameter constraint. The diameter of each pipe must belong to the set of optional standard diameters.

The seventh constraint is looped pipe network constraint. All the pipelines in connection status should be a looped pipe network.

The final constraint is water supply constraint. All the water positions can be supplied with water. 
Through the designs of coding and operation of the algorithm, only the node pressure constraint can't be satisfied directly, the others constraints are satisfied automatically. Finally, through the penalty function method, the final objective function is formed, that is

$$
f=f_{3}+M \sum_{i=1}^{N_{\mathrm{n}}} \max \left(0, p_{i \min }-p_{i}\right)
$$

In the type, $M$ is penalty parameters of pressure, $N_{\mathrm{n}}$ is the total number of water positions.

\section{Improved Genetic Particle Swarm Optimization Algorithm}

Coding Design. In the design variables, the start-up and stop status of pump and the connection status of pipeline can be expressed by 0 or 1 , flow rate of pump is continuous variable, and diameter of pipeline is discrete variable and normalized value.

Binary coding is adopted to express the variables 0 and 1 . In order to improve the accuracy of calculation, real number coding is adopted to express the flow rate. Integer coding is adopted to express the diameter. If there are $N_{\mathrm{sl}}$ kinds of diameter, an integer from 1 to $N_{\mathrm{sl}}$ corresponds to one diameter.

Based on the generalized chromosome coding method[17], dual generalized chromosome coding is designed. Optimal variables about pump in the left side of chromosome, and Optimal variables about pipeline in the right side of chromosome. In the left side, the binary coding is adopted to express the start-up and stop status of pump in the first line, the real number coding is adopted to express the flow rate in the second line, the length of coding is equal to $N_{\mathrm{p}}$. In the right side, the binary coding is adopted to express the connection status of pipeline in the first line, the integer coding is adopted to express the diameter in the second line, the length of coding is equal to $N_{\mathrm{L}}$. An example of coding is shown in Figure 1.

$\begin{array}{cccc:cccccc}0 & 1 & 1 & 0 & 1 & 0 & 1 & 1 & 1 & 0 \\ 312.5 & 298 . & 325.4 & 357.8 & 3 & 1 & 2 & 4 & 2 & 1\end{array}$

Fig. 1 The diagram of dual generalized chromosome coding

Generation of Initial Population. In order to avoid the generation of infeasible solutions, generation methods of initial population are designed.

For the pump coding, the binary coding is generated firstly, and its feasibility judgment is needed. The sums of minimum and maximum flow rate of running pump are computed respectively, the water requirement of the system should be in the range. If the binary coding is not feasible, the coding needs to be regenerated. Then the real number coding is generated. The value of flow rate should be in the range $\left[Q_{i \min }, Q_{i \max }\right.$, and the sum of flow rates of all running pumps is equal to the total water requirement of the system, so the water balance constraint can be satisfied.

For the pipeline coding, the binary coding is generated firstly. In order to reduce the number of infeasible solutions, the related knowledge of graph theory is used. The cycle can be divided into two kinds, the first kind cycle is the closed circuit whose starting point and end point are the same one, and the second kind cycle only aims at multi-source system, the starting point and the end point are different source. The generating method of looped pipe network can be divided into two steps. Firstly, the binary coding is assigned to 1, i.e. all the pipelines are in connection status, forming the preliminary connection graph. Secondly, randomly select partial cycles, and judge whether disconnected pipelines exist in those cycles, if not, disconnect any pipeline in the cycles randomly, and ensure there are least one cycle in the graph. The ultimate network is a looped network, and all the nodes in the network can be supplied with water. For the integer coding, let the gene value equal to a random integer in the range from 1 to $N_{\mathrm{sl}}$.

Crossover Operation. We design a variety of crossover methods, and select one method randomly. 1) Binary coding crossover of pump. We select two parent individuals that their genes of binary coding are different, single point crossover or coding interchange is adopted randomly, the essence of the crossover method is to change the start-up and stop status of pump. After the 
crossover, the flow rates of all running pumps need to be adjusted to meet the water balance constraint. 2) Real number coding crossover of pump. The arithmetic crossover method is adopted. If the start-up and stop statuses of pumps are the same in the two parent individuals, the flow rates are not need to be adjusted after the crossover. If the running statuses of pumps are different, the flow rates of all running pumps need to be adjusted. 3) Binary coding crossover of pipeline. We select two parent individuals that their genes of binary coding of pumps are the same, coding interchange or single point crossover is adopted randomly. If the single point crossover is adopted, we should determine whether the looped pipe network constraint and water supply constraint are satisfied, if not, the adjustment of the binary coding of pipeline is required. If the coding interchange is adopted, the adjustment is not required. 4) Integer coding crossover of pipeline. The arithmetic crossover method is adopted, and the decimal should be rounded off. Pipe diameter constraint is satisfied automatically.

Mutation Operation. We design four kinds of mutation methods. 1) Binary coding mutation of pump. One gene is selected randomly, and the gene value is changed from 1 or 0 to 0 or 1 , i.e. the number of running pumps is decreased or increased by 1 . After the mutation, the feasibility of the binary coding should be judged, and the flow rates of all running pumps should be adjusted. 2) Real number coding mutation of pump. Two genes that their binary coding values are equal to 1 are selected randomly, one gene value is increased a small value, and another gene value is decreased the same value. In this operation, the infeasible solutions are avoided. 3) Binary coding mutation of pipeline. Randomly select one disconnected pipeline (the gene value is 0 ), and change the status into connection status (the gene value change into 1), i.e. the pipeline number of connection status is increased by 1 , so the pipe network is still a looped pipe network. If the gene value is changed from 1 or 0 , we should select one coding, and in the network corresponding to the coding must have at least two cycles. This operation can greatly improve the probability of generating feasible solutions. 4) Integer coding mutation of pipeline. One gene vale is increased or decreased a small integer randomly within the limits of diameter constraint, and the infeasible solutions are avoided.

Real Number Optimization Using Particle Swarm Optimization Algorithm. In order to improve the local searching ability for continuous variable, partial chromosomes are selected randomly, and the discrete variables remain fixed, the particle swarm optimization algorithm [11] is adopted to optimize the flow rates.

For each chromosome, the flow rates of running pumps are optimization variables. We assume that there are $M$ particles with $N_{\text {rp }}$ dimension corresponding to one problem. $N_{\text {гр }}$ is the number of running pumps, i.e. the number of 1 in the binary coding of pump. $\mathbf{v}_{i}=\left(v_{i 1}, v_{i 2}, \cdots, v_{i d}, \cdots, v_{i N_{\mathrm{rp}}}\right)$ is the velocity of the particle $i$ in the $N_{\mathrm{rp}}$ dimension, $\mathbf{x}_{i}=\left(x_{i 1}, x_{i 2}, \cdots, x_{i d}, \cdots, x_{i \mathrm{~N}_{\mathrm{rp}}}\right)$ is the position of the particle $i$ in the $N_{\text {rp }}$ dimension. The update speed and position could be expressed as

$$
\begin{aligned}
& v_{i d}^{k}=\omega v_{i d}^{k-1}+c_{1} \operatorname{rand}_{1}\left(p_{i d}-x_{i d}^{k-1}\right)+c_{2} \operatorname{rand}_{2}\left(p_{g d}-x_{i d}^{k-1}\right) \\
& x_{i d}^{k}=x_{i d}^{k-1}+v_{i d}^{k-1}
\end{aligned}
$$

In the type, $k$ is number of Iterations, $\omega$ is the inertial weight, $\omega=1.0, c_{1}$ and $c_{2}$ are convergent factors, $c_{1}=c_{2}=2.0$, rand $d_{1}$ and $\operatorname{rand}_{2}$ are random functions generating a number in the range [0,1], $p_{i}$ is the best previous position of the particle $i, p_{g}$ is the best particle so far among all the particles, $d$ is the dimension in the search space.

\section{Optimization Example}

To validate the algorithm proposed above, we optimize a water injection system. The preliminary connection graph of the looped pipe network is shown in Figure 2. There are 15 nodes and 20 pipelines, 2 water injection stations and 11 consumption nodes. The unit of flow rate is L/s. The detailed results of after optimization are shown in Table 1 and Table 2. 


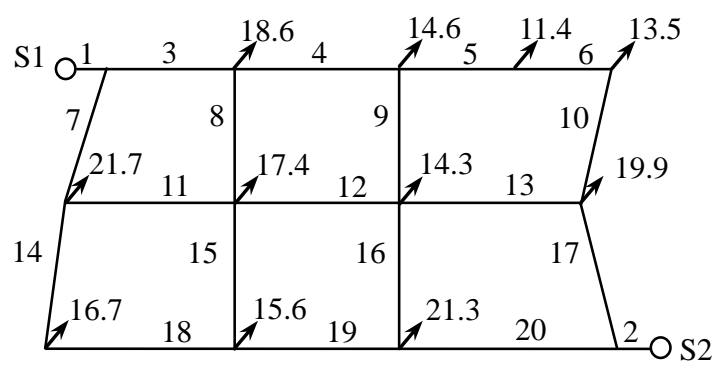

Fig.2 The preliminary connection graph of the pipe network

Tab.1 The comparison of pump station operation schemes

\begin{tabular}{ccccccc}
\hline & & $\begin{array}{c}\text { Running } \\
\text { pump } \\
\text { number }\end{array}$ & $\begin{array}{c}\text { Flow } \\
\text { rate } \\
(\mathrm{L} / \mathrm{s})\end{array}$ & $\begin{array}{c}\text { Head of } \\
\text { pump } \\
(\mathrm{MPa})\end{array}$ & $\begin{array}{c}\text { Efficiency } \\
\text { of pump } \\
(\%)\end{array}$ & $\begin{array}{c}\text { Energy } \\
\text { consumption } \\
(\mathrm{kW})\end{array}$ \\
\hline $\begin{array}{c}\text { Current } \\
\text { operation } \\
\text { state }\end{array}$ & $\begin{array}{c}\text { The first } \\
\text { station } \\
\text { The second } \\
\text { station }\end{array}$ & 2 & 90.4 & 16.7 & 80.62 & 1950 \\
\hline $\begin{array}{c}\text { After } \\
\text { optimization }\end{array}$ & $\begin{array}{c}\text { The first } \\
\text { station } \\
\text { The second } \\
\text { station }\end{array}$ & 1 & 94.6 & 16.75 & 78.70 & 2096 \\
\hline
\end{tabular}

Tab.2 The comparison of pipe network design schemes

\begin{tabular}{cccccc}
\hline \multirow{2}{*}{ Pipe number } & $\begin{array}{c}\text { Pipe length } \\
(\mathrm{m})\end{array}$ & \multicolumn{2}{c}{ Current operation state } & \multicolumn{2}{c}{ After optimization } \\
\cline { 3 - 6 } & 100 & 1 & 245 & 1 & 273 \\
\hline 1 & 150 & 1 & 245 & 1 & 273 \\
2 & 894 & 1 & 219 & 1 & 245 \\
3 & 1044 & 1 & 219 & 1 & 219 \\
4 & 884 & 1 & 219 & 1 & 168 \\
5 & 859 & 1 & 168 & 1 & 219 \\
6 & 1115 & 1 & 219 & 1 & 245 \\
7 & 1000 & 0 & - & 0 & - \\
8 & 991 & 0 & - & 0 & 219 \\
9 & 1169 & 1 & 219 & 1 & 168 \\
10 & 1371 & 1 & 219 & 0 & - \\
11 & 1189 & 1 & 168 & 1 & 219 \\
12 & 1061 & 1 & 219 & 1 & 219 \\
13 & 1034 & 1 & 219 & 1 & 168 \\
14 & 928 & 0 & - & 0 & - \\
15 & 981 & 0 & - & 1 & 245 \\
16 & 936 & 1 & 219 & 0 & 219 \\
17 & 1568 & 1 & 168 & 1 & 219 \\
18 & 1247 & 1 & 219 & 1 & \\
19 & 1354 & 1 & 219 & $3857 \mathrm{~kW}$
\end{tabular}

After optimization, the total input power from $4046 \mathrm{~kW}$ reduced to $3857 \mathrm{~kW}$, reduced by $4.67 \%$. The optimized effect is better. The total investment of pipe network decrease from 11.554 million Yuan to 10.484 million Yuan, the total investment is saved 9.26\%. The optimized effect is better.

\section{Conclusion}

In order to achieve the best running status of the water injection system, the simultaneous optimization of the start up scheme and pipe network is proposed, the optimization objective function considering the energy consumption and investment is established. The start-up and stop status, flow rate of pump, and the connection status, diameter of pipeline are optimized 
simultaneously.

Aiming at the features of the simultaneous optimization problem, a dual generalized chromosome coding was designed, and the particle swarm optimization algorithm is combined with the genetic algorithm. The solution procedures of the improved algorithm include coding, crossover and mutation are designed, and some constraints are satisfied automatically, the optimization efficiency of the algorithm is increased.

\section{Acknowledgement}

In this paper, the research was sponsored by the Project of Shandong Province Higher Educational Science and Technology Program (Project No. J13LB13).

\section{References}

[1] Broad D R, Maier H R, Dandy G C, Asce M. Optimal operation of complex water distribution systems using metamodels[J]. Journal of Water Resources Planning and Management, 2010, 136(4):433-443.

[2] Vieira J, Cunha M C, Nunes L, Monteiro J P, Ribeiro L, Stigter T, Nascimento J, Lucas H. Optimization of the operation of large-scale multisource water-supply systems[J]. Journal of Water Resources Planning and Management, 2011, 137(2): 150-161.

[3] Zhou L C. Operation optimization of the pumping station with multiple parallel-pumps units[J]. Journal of Irrigation and Drainage,2014,33(1):96-99.

[4] Yang J J, Liu Y, Wei L X, Zhan H. Dual coding hybrid genetic algorithm for optimal schedule of pumping stations in multi-sources water injection system[J]. Acta Automatica Sinica, 2006,32(1):154-160.

[5] Zheng F F, Simpson A R, Zecchin A C. Dynamically expanding choice-table approach to genetic algorithm optimization of water distribution systems [J]. Journal of Water Resources Planning and Management, 2011,137(6):547-551.

[6] Mohan S, Babu K S. Optimal water distribution network design with honey-bee mating optimization[J]. Journal of Computing in Civil Engineering, 2010,24(1):117-126.

[7] Vasan A, Simonovic S P. Optimization of water distribution network design using differential evolution [J]. Journal of Water Resources Planning and Management, 2010,136(2):279-287.

[8] Qiao J F, Wang C, Liu C F. Optimal design of a water supply system based on improved self-adaptive particle swarm algorithm[J]. Journal of Beijing University of Technology, 2014,40(7):1035-1040.

[9] Geen Z W. Harmony search optimisation to the pump-included water distribution network

design[J]. Civil Engineering and Environmental Systems, 2009,26(3):211-221.

[10] Wu W Y, Simpson A R, Maier H R. Accounting for greenhouse gas emissions in multiobjective genetic algorithm optimization of water distribution systems [J]. Journal of Water Resources Planning and Management, 2010,136(2):146-155.

[11] Kennedy J, Eberhart R. Particle Swarm Optimization [C], Proc. IEEE Int. Conf. Neural Networks, 1995,4:1942-1948. 